
\title{
Chemotherapeutic Toxicity And Symptom Management In Geriatric Patients With Cancer
}

\author{
Nurhan Doğan* and Kenan Gümüş \\ Department of Nursing, Amasya University, Turkey
}

Received: December 19, 2018; Accepted: February 16, 2018; Published: February 23, 2018

*Corresponding author: Dr. Nurhan Doğan, Department of Nursing, Faculty of Health Sciences, Amasya University, Turkey, Tel: +90358 21817 67; Fax: +90358 21847 60; E-mail:nurhan_dogan38@hotmail.com

\begin{abstract}
Introduction: It is very important to perform a comprehensive geriatric assessment to prevent the development of physiological and psychological complications that may be associated with cancer and treatment in elderly individuals.

Method: Literature review was presented extracting from the full texts and summarized articles reached via databases such as "Pub Med, Scopus, Scince Direct, Ulakbim, Google Scholar" by using key words such as chemotherapeutic toxicity, symptom management, geriatric patients and cancer.

Results: Complications such as myelosuppression, gastrointestinal toxicity, fluid electrolyte imbalance, metabolic problems, skin reactions, ulcers, photosensitivity, organ toxicity are very common in comoterapy. In order to control these complications, which may be seen more aggressively in elderly individuals, the patient, family and health personnel should work as a whole.

Conclusion: Routinely informed about comprehensive geriatric assessment, disease status, functional capacity and general health risks, quality of life should be kept at maximum level. Whenever possible, the person performing the patient should protect their activities independently. Individual and family cancer treatment procedures and chemotherapy complications and symptom management should be trained.
\end{abstract}

Keywords: Cancer; Chemotherapeutic Toxicity; Geriatric Patients; Symptom Management

\section{Introduction}

Innovation in health care technology, the expansion of health services to individuals prolonged life expectancy has increased with the raising of awareness and the elderly population [1-3]. Along with age progression, the incidence of cancer is increasing, but cancer-related deaths are also increasing. According to statistics and studies done, the incidence of cancer and the majority of cancer deaths were seen in individuals over 65 years of age [3-8]. The causes of cancer-related deaths in the elderly include inadequate anti-tumoral treatment, inability to tolerate treatment, lack of age-specific treatment protocols, reduction in functional capacity, psychological, social and comorbid health problems and inadequate use of preventive methodsIt is very important to make comprehensive geriatric assessment to prevent the development of physiological and psychological complications that may be associated with cancer and treatment in elderly individuals, because in these patients the treatment gain is minimal and the treatment toxicity is high [3, 7- 9]. The greatest concern during the use of chemotherapeutic drugs used in cancer treatment is toxicity. Toxicities of these drugs need to be known and monitored $[8,10]$. In elderly patients, due to age-related physiological changes, the mechanism of action of the drug, the dosage and form of administration, the pharmacokinetics and pharmacodynamics of the drug, the protocol of drug or drug administration, the adequacy of the person's liver, kidney and bone marrow functions, co-morbidity and has been spread to bone marrow of the disease, toxicity is determined [8; 10-13]. It is vital that geriatric cancer is vital in the development of cancer, because individuals are able to tolerate treatment, have correctable conditions that may affect treatment, management of treatment symptoms, increase in patient's quality of life, and individual differences in the aging process $[3,8,12,14-16]$. The elderly individual should be treated with necessary psycho and / or pharmacotherapeutic approaches and caregivers should be ensured. Many causes such as depression, functional and cognitive disorders lead to malnutrition. Individuals should be closely monitored for malnutrition, polypharmacy status should be learned and drug interaction should be assessed. For renally withdrawn drugs, dose adjustment should be done by calculating glomerular filtration rate. Chemotherapeutic toxicities; myelosuppression (bone marrow toxicity), gastrointestinal toxicity (nausea-vomiting, anorexia, oral mucositis, diarrhea, constipation), fluid-electrolyte imbalance, metabolic problems (such as impaired glucose regulation), skin reactions (dryness, pruritus, erythema, urticaria, ulcer, photosensitivity, alopecia), organ toxicity (hepatotoxicity, pulmonary toxicity, cardiac toxicity, neurotoxicity, nephrotoxicity) in terms of individuals should be monitored closely $[12,13,17,18]$.

\section{Method}

Literature review was presented extracting from the full texts and summarized articles reached via databases such as "Pub Med, Scopus, Scince Direct, Ulakbim, Google Scholar" by using key words such as chemotherapeutic toxicity, symptom management, geriatric patients and cancer. The review was carried out on the magazines which can only be accessed on-line in the screening process. 


\section{Result}

The recognition and reporting of side effects of chemotherapy in geriatric patients is at least as important as the efficacy of the treatment; but it is reported that there is not yet a standard developed in the world in this respect, but the question of side effects and the quality of life findings are behind the scenes. The development of tools to measure the individual's risk of serious toxicity from chemotherapy and these tools are thought to be of considerable benefit, especially for older patients [Table 1].

\begin{tabular}{|c|c|}
\hline Myelosuppression & $\begin{array}{l}\text { Bone marrow toxicity (neutropenia, } \\
\text { thrombocytopenia, anemia) }\end{array}$ \\
\hline \multirow{5}{*}{ Gastrointestinal toxicity } & Nausea-vomiting \\
\hline & Anorexia \\
\hline & Oral mucositis \\
\hline & Diarrhea \\
\hline & Constipation \\
\hline \multicolumn{2}{|c|}{ Fluid-electrolyte imbalance } \\
\hline Metabolic problems & İmpaired glucose regulation \\
\hline \multirow{7}{*}{ Skin reactions } & Dryness \\
\hline & Pruritus \\
\hline & Erythema \\
\hline & Urticaria \\
\hline & Ulcer \\
\hline & Photosensitivity \\
\hline & Alopecia \\
\hline \multirow{5}{*}{ Organ toxicity } & Hepatotoxicity \\
\hline & Pulmonary toxicity \\
\hline & Cardiac toxicity \\
\hline & Neurotoicity \\
\hline & Nephrotoxicity \\
\hline
\end{tabular}

\section{References}

1. Biçer S. Health Problems in Elderly People and Nursing Approaches. In: Ovayolu N, Ovayolu Ö. (Editors), Basic Internal Diseases Nursing and Chronic Diseases with Different Dimensions. Turkey. Adana Nobel Bookstore, Adana. 2016.

2. Beğer T, Yavuzer H. Old age and old age epidemiology. Journal of Clinical Development. 2012; 25: 1-3.

3. Tuna S. Comorbidity and clinical assessment in geriatric patients with cancer. Turkish Journal of Oncology. 2007; 22(4):192-196.
4. Jemal A, Bray F, Center MM, Ferlay J, Ward E, Forman D. Global cancer statistics. CA Cancer J Clin. 2011; 61(2):69-90. Doi: 10.3322/ caac.20107

5. Siegel RL, Miller KD, Jemal A. Cancer Statistics, 2015. CA Cancer J Clin. 2015; 65(1):5-29. Doi: 10.3322/caac

6. Alan Ö, Gürsel Ö, Ünsal M, Altın S, Kılçıksız S. Oncologic Approach to Geriatric Patients. Okmeydanı Medical Journal. 2013; 29(2):94-98.

7. Mohile SG, Klepin HD, Rao AV. Considerations and controversies in the management of older patients with advanced cancer. Am Soc Clin Oncol Educ Book. 2012; 321-328. Doi: 10.14694/EdBook_AM.2012.32.321

8. Bostankolu Ö, Öztürk B, Coşkun U, Büyükberber S, Benekli M. Cancer Chemotherapy in Elderly Patients. International Journal of Hematology and Oncology. 2008; 3(18): 186-192.

9. Savaș S, Akçiçek F. Comprehensive geriatric assessment. Ege Journal of Medicine. 2010; 49(3)Supplement: 19-30.

10. Chatelut E, Delord JP, Canal P. Toxicity patterns of cytotoxic drugs. Invest New Drugs. 2003; 21(2):141-148.

11. Üreyen I, Karalök A, Hızlı D, Tohma A, Tapısız Ö. Age-related effect on hematological toxicity in the first-line adjuvant group receiving paclitaxel / carboplatin chemotherapy. New Medical Journal. 2012. 29(4):202-205.

12. Chen H, Cantor A, Meyer J, Beth Corcoran M, Grendys E, Cavanaugh D, Et al. Can older cancer patients tolerate chemotherapy? A prospective pilot study. Cancer. 2003; 15; 97(4):1107-1114. Doi:10.1002/ cncr.11110

13. Hurria A, Lichtman SM. Pharmacokinetics of chemotherapy in the older patient. Cancer Control. 2007; 14(1):32-43. Doi:10.1177/107327480701400105

14. Scharf O, Colevas AD. Adverse event reporting in publications compared with sponsor database for cancer clinical trials. J Clin Oncol. 2006; 24(24): 3933-3938. Doi:10.1200/JC0.2005.05.3959

15. Gosney MA. Clinical assessment of elderly people with cancer. Lancet Oncol. 2005; 6(10):790-797. Doi: 10.1016/S1470-2045(05)70389-2

16. Extermann M, Boler I, Reich RR, Lyman GH, Brown RH, DeFelice J, Et al. Predicting the risk of chemotherapy toxicity in older patients: the Chemotherapy Risk Assessment Scale for High-Age Patients (CRASH) score. Cancer. 2012; 118(13):3377-86. Doi: 10.1002/cncr.26646.

17. Lichtman SM, Wildiers H, Launay-Vacher V, Steer C, Chatelut E, Aapro M. International Society of geriatric Oncology (SIOG) recommendations for the adjustment of dosing in elderly cancer patients with renal insufficiency. Eur J Cancer. 2007; 43(1):14-34. Doi:10.1016/j. ejca.2006.11.004

18. Atasoy BM, Ünsal DK, Dinçbaş FÖ, Yamaç D, Okkan S, İçli F. Questionnaire results on side effect assessment and enrollment: "The Turkish oncology group (TOG) early-late side effects study group" survey. Turkish Journal of Oncology. 2007; 22(1): 13-19.

19. Erdoğan T, Tunca H. Multifunctional functional assessment of geriatric patients attending to internal medicine polyclinic and examination of nutritional status. Osmangazi Journal of Medicine. 2016; 38: 1-8. 